\title{
Relations between the sensory properties and fat ingredients of lipsticks
}

\author{
Hélène de Clermont-Gallerande ${ }^{1, *}$, Sarah Abidh $^{1,2}$, Alexandre Lauer ${ }^{1}$, Séverine Navarro ${ }^{1}$, \\ Gérard Cuvelier ${ }^{2}$ and Julien Delarue ${ }^{2}$ \\ ${ }^{1}$ Chanel Parfums Beauté, Recherche Beauté et Innovation, 93500 Pantin, France \\ ${ }^{2}$ UMR Ingénierie Procédés Aliments, AgroParisTech, Université Paris-Saclay, 91300 Massy, France
}

Received 26 April 2018 - Accepted 14 September 2018

\begin{abstract}
Lipstick is a key product in the make-up sector. A woman applies lipstick to feel feminine and attractive. The sensation she perceives when she applies the product plays an important role in her attachment to her lipstick. The impact of the ingredients on the sensory properties and the quality of the lipsticks needs to be understood, so that the formulation can be more effective and the sensory properties can be precisely adjusted to the target market. During this study, multidimensional correlations were made between the percentage of ingredients, their physico-chemical specifications and the sensory properties of the raw materials on their own or the lipsticks. The objective of this study is to predict the sensory properties of lipsticks from the physico-chemical specifications of the ingredients. It is in effect quicker to access the physical data than the sensory descriptions. The lipsticks were made using a simplified formula, evaluated in a sensory analysis and their physico-chemical characteristics were measured. The relationships between the sensory properties, the ingredient specifications and their percentage of use in the formula were highlighted. The results confirm the major role of the viscosity of oils and the wax used in the formula on the sensory and mechanical properties of the lipstick. It is therefore possible to modify the sensory properties, for example to adjust the shininess of a lipstick, without altering its mechanical resistance. This opens up opportunities for developing innovative sensory textures in short lead times.
\end{abstract}

Keywords: lipstick / sensory properties / oils / waxes / formulation

Résumé - Relations entre les propriétés sensorielles et les ingrédients gras des rouges à lèvres. L'objectif de cette étude est de prédire la sensorialité des rouges à lèvres à partir des spécifications physicochimiques des ingrédients. En effet, les données physiques sont plus rapidement accessibles que les descriptions sensorielles. À partir d'une formule simplifiée, des rouges à lèvres sont formulés, évalués en analyse sensorielle et leurs caractéristiques physico-chimiques sont mesurées. Les relations entre les propriétés sensorielles, les spécifications des ingrédients et leur pourcentage d'introduction dans la formule sont mises en évidence. Les résultats confirment le rôle majeur de la viscosité des huiles et de la cire sélectionnée dans la formule sur les propriétés sensorielles et mécaniques du rouge à lèvres. Il est ainsi possible de modifier des propriétés sensorielles, par exemple, ajuster la brillance d'un rouge à lèvres, sans en changer sa résistance mécanique. Cela ouvre des opportunités pour développer des textures sensoriellement innovantes dans un délai plus restreint.

Mots clés : rouge à lèvres / propriétés sensorielles / huiles / cires / formulation

\footnotetext{
*Correspondence:

helene.declermontgallerande@chanel-corp.com
} 


\section{Introduction}

France is the world leader in cosmetics, with high export sales figures. In the competitive market of luxury make-up brands, lipstick is a key product as it represents $8.5 \%$ of these sales ${ }^{1}$. Today, lipstick is a flagship make-up product with over 900 million units sold per year worldwide, 300 million in Europe $^{2}$. It is the best-selling cosmetic product. Several journalists have remarked that in a time of economic crisis, sales of premium lipsticks seem to rise (Nelson, 2001; Schaefer, 2008; Martinez and Allison, 2010). This notion has been nicknamed the "lipstick effect" (Hill et al., 2012). Lipstick can also be a morale booster, as it creates positive emotions such as attractiveness, self-confidence and femininity (Russell et al., 2016). Lipstick is also a highly adaptable product. A woman might buy on average up to six lipsticks a year, either to replace a used lipstick or to change her style (Oglivie and Kristensen-Bach, 2015). Lipstick is also a small object, easy to carry in her handbag and easy to apply. There are however rules about its application, which is usually done "in private". It is an intimate gesture and moment for women, probably linked to its impact on her self-confidence and selfesteem. Although a woman will buy a lipstick according to the colour and the make-up effect, she will only buy the same one again if she is attracted by the sensory nature of the product. Sensory properties such as slipperiness, smoothness, thickness of the coating, and a moisturising sensation are strategic criteria in repeating the purchase.

When she makes a purchase, she expects to see a "beautiful, perfectly smooth lipstick with no defects". The visual aspect is one quality criteria of lipsticks. The consumer will then apply the lipstick to her lips and it must not break when she uses it. Resistance to breaking is also a quality criterion. During application, the lipstick must meet a number of sensory expectations linked to the sensation on the lips and the appearance of the lipstick coating just after application. The sensory properties of the lipstick are therefore important to consumers and a crucial factor in the choice of product (Desmet and Hekkert, 2007). Every woman, depending on her culture, personality and education, expects a specific make-up effect. To meet these demands and although the range of lipstick textures is already very diverse, cosmetic companies are exploring new sensory territories. Chanel offers five lines of lipsticks with different make-up effects (classic, matte, shiny, long-lasting, transparent) to meet women's expectations all over the world. The Rouge Coco range from Chanel PB has 33 different shades of lipstick, to reach a target market that is as wide as possible. Other shades may appear from time to time depending on the season; these are known as collections.

During this study, we acquired knowledge about the relations between the ingredients in the formula and the

\footnotetext{
${ }^{1}$ Fédération des entreprises de la Beauté. Available from http://www. febea.fr/fr/le-secteur-cosmetique/le-secteur-chiffres (last consult 2018/02/02)

${ }^{2}$ ConsoGlobePlanetoscope-Statistiques: ventes mondiales de rouges à lèvres. Available from https://www.planetoscope.com/ hygiene-beaute/1302-nombre-de-rouge-a-levres-vendus-en-europe. html (last consult 2018/02/02).
}

sensory characteristics of the lipstick. We investigated the role of oils and waxes on the sensory properties of the lipsticks. In particular, we studied the impact of the physico-chemical properties of oils, such as viscosity and the refractive index, and their sensory properties such as the slipperiness and shininess of the lipsticks. We also compared the impact of the variation in quantity and the chemical nature of the wax in three different formulas of lipstick, on the sensory properties of these lipsticks. The quality of the lipsticks was tested in all the tests by measuring the breaking index. The objectives of this study are:

- to provide knowledge on how to formulate a lipstick with defined sensory properties, so that Chanel can meet the expectations of consumers all over the world.

- to extend the range to new sensory territories, while maintaining the selective quality of luxury products and reducing development time for lipsticks.

\section{Material and methods}

\subsection{Lipsticks}

Ingredients: oils

The INCI names and the physico-chemical specifications of the oils used in the study are given in Table 1 .

Ingredients: wax

The INCI name of the wax used is synthetic wax \& polyethylene \& polypropylene/ethylene copolymer. Its melting point is $84{ }^{\circ} \mathrm{C} \pm 2{ }^{\circ} \mathrm{C}$ and its enthalpy of fusion is $200 \pm 10 \mathrm{~J} / \mathrm{g}$.

\section{Lipsticks: operating method}

One kilogram of lipsticks is made in a laboratory. The waxes and oils were weighed and mixed together before being heated and slowly stirred at $98 \pm 2{ }^{\circ} \mathrm{C}$. The pigments were added once the mixture had completely melted. After homogenisation, the mixture was poured into silicone moulds at $90{ }^{\circ} \mathrm{C} \pm 2{ }^{\circ} \mathrm{C}$. The mixture was cooled to $0{ }^{\circ} \mathrm{C} \pm 2{ }^{\circ} \mathrm{C}$ for 10 minutes before extraction. After 15 days of maturation at $20^{\circ} \mathrm{C}$, physico-chemical measurements and sensory evaluations were carried out on the lipsticks.

Lipsticks are made of a complex anhydrous mixture that may contain up to 30 raw materials / ingredients (de ClermontGallerande, 2006). In this study, the pigments and powders are fixed as the experiments focus only on fatty ingredients, more specifically on oils and waxes and their impact on the sensory properties of the product. The use of previous studies and expert knowledge helped us to draw hypotheses on the supposed impact of the wax and oils on the sensory functions of the lipsticks (Savary et al., 2013).

The oils, which are mainly characterized by their viscosity and refractive index as well as their sensory properties, are known to impact the slipperiness of lipsticks (Abidh, 2017) and are believed to impact the shininess of lipsticks.

The waxes, which are mainly characterized by their melting properties, are supposed to impact the long-lasting properties, lip definition and thickness of the lipsticks.

In this study, we chose to investigate first the impact of the viscosity and the refractive index of the oils on the shininess of the lipsticks, and the relationship between the sensory properties of the oils and the sensory properties of the 
Table 1. Measures of Refractive Index. Glossiness and viscosity of several cosmetic oils.

\begin{tabular}{llll}
\hline Oils & Refractive Index $\left(20^{\circ} \mathrm{C}\right)$ & Glossiness SAMBA & Viscosity Pa.s $\left(20^{\circ} \mathrm{C}\right)$ \\
\hline Dicaprylyl carbonate & 1.4345 & 389.80 & $0.01050 \pm 0.00004$ \\
Isononyl isononanoate & 1.4350 & 392.50 & $0.0090 \pm 0.00006$ \\
Octyldodecyl neopentanoate & 1.4450 & 401.20 & $0.0210 \pm 0.00010$ \\
Caprylic/capric triglyceride & 1.4500 & 407.40 & $0.0305 \pm 0.00010$ \\
Pentaerythrityl tetraoctanoate & 1.4520 & 410.60 & $0.1400 \pm 0.00200$ \\
Octyl dodecanol & 1.4535 & 361.30 & $0.0580 \pm 0.00040$ \\
Hydrogenated polyisobutene & 1.4550 & 418.30 & $0.0490 \pm 0.00010$ \\
Squalane & 1.4555 & 415.70 & $0.0490 \pm 0.00010$ \\
Hydrogenated polydecene & 1.4595 & 420.20 & $0.0700 \pm 0.00010$ \\
Diisostearyl malate & 1.4610 & 421.70 & $5.3020 \pm 0.02800$ \\
Polyester-4 & 1.4655 & 427.40 & $1.1650 \pm 0.00900$ \\
Jojoba oil & 1.4660 & $\mathrm{NA}$ & $0.0430 \pm 0.00060$ \\
Polyglyceryl-2 triisostearate & 1.4675 & 430.11 & $0.5490 \pm 0.00300$ \\
Meadowfoam seed oil (Limnanthes alba) & 1.4710 & 398.00 & $0.1040 \pm 0.00200$ \\
Polyglyceryl-10 nonaisostearate & 1.4715 & 435.60 & $1.8390 \pm 0.01200$ \\
Phytosteryl/octyldodecanol lauroyl glutamate & 1.4800 & 443.90 & $2.9740 \pm 0.03400$ \\
Polybutene & 1.4960 & 466.50 & Too thick \\
Phenylpropyldimethylsiloxysilicate & 1.5155 & 498.70 & $1.2010 \pm 0.01100$ \\
\hline
\end{tabular}

lipsticks. We then studied the impact of the quantity of wax on the thickness of the lipsticks.

Using a simple but realistic formula developed in a previous study (Abidh et al., 2016), we performed two experiments:

- the sensory and physical properties of 14 oils were evaluated and the relationships between the two sets of properties were established. Of those 14 oils, six were chosen to formulate six different lipsticks;

- the wax was varied in quantity in three different environments (three different viscosities of oils used in the previous experiment).

The quality of the lipsticks was assessed according to the measurement of their resistance to breaking.

\subsection{Sensory analysis (Abidh, 2017)}

There are many sensory analysis methods applicable to cosmetic products, which are summed up by Pensé-Lhéritier (2015). The industry generally uses quantitative descriptive sensory profile methods, such as the Spectrum ${ }^{(M)}$ (Meilgaard et al., 2006) method or a variant. Information relating to the sensory analysis of lipstick is confidential and virtually absent from the literature. However, Dooley et al. (2009) have developed and published a lexicon for the sensory analysis of lip products. The lexicon contains 16 criteria, measured during or after application, and placed in four groups: initial texture, initial appearance, after texture and after appearance. The two "after application" groups correspond to an assessment 10 minutes after applying the product. An order and a technical evaluation are associated with each of the criteria. These give a good overview of the sensory expectations, texture and appearance of a lip product. However, this lexicon has been

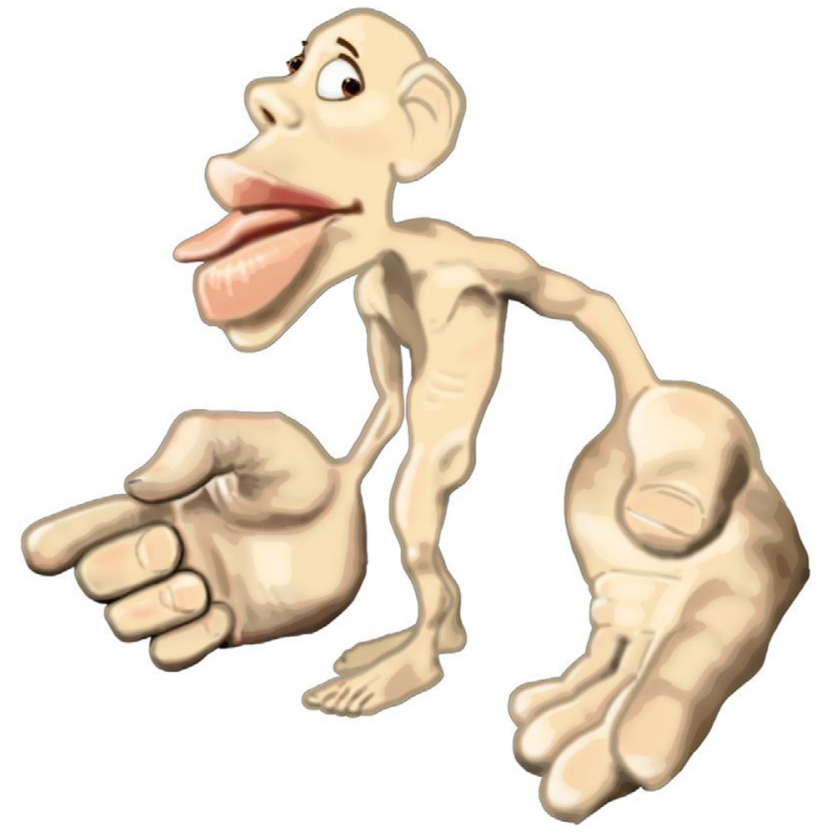

Fig. 1. The sensory homunculus or cortical homunculus from Dr. Wilder Penfield.

introduced for applying the lip product to the forearm, and requires several applications of the product. An application on the forearm enables more products to be evaluated at the same time, as the available surface area is larger than the lips. However, there are not as many sensory receptors on the forearm as on the lips, as can be seen in Figure 1. The sensory homunculus also known as cortical homunculus represents the parts of the body in proportion to the part of the brain receiving the sensory nerves coming from the different parts. A larger 
Table 2. Lip products sensory evaluation protocol, including the criteria definitions, drawn up by the CHANEL PB sensory analysis department.

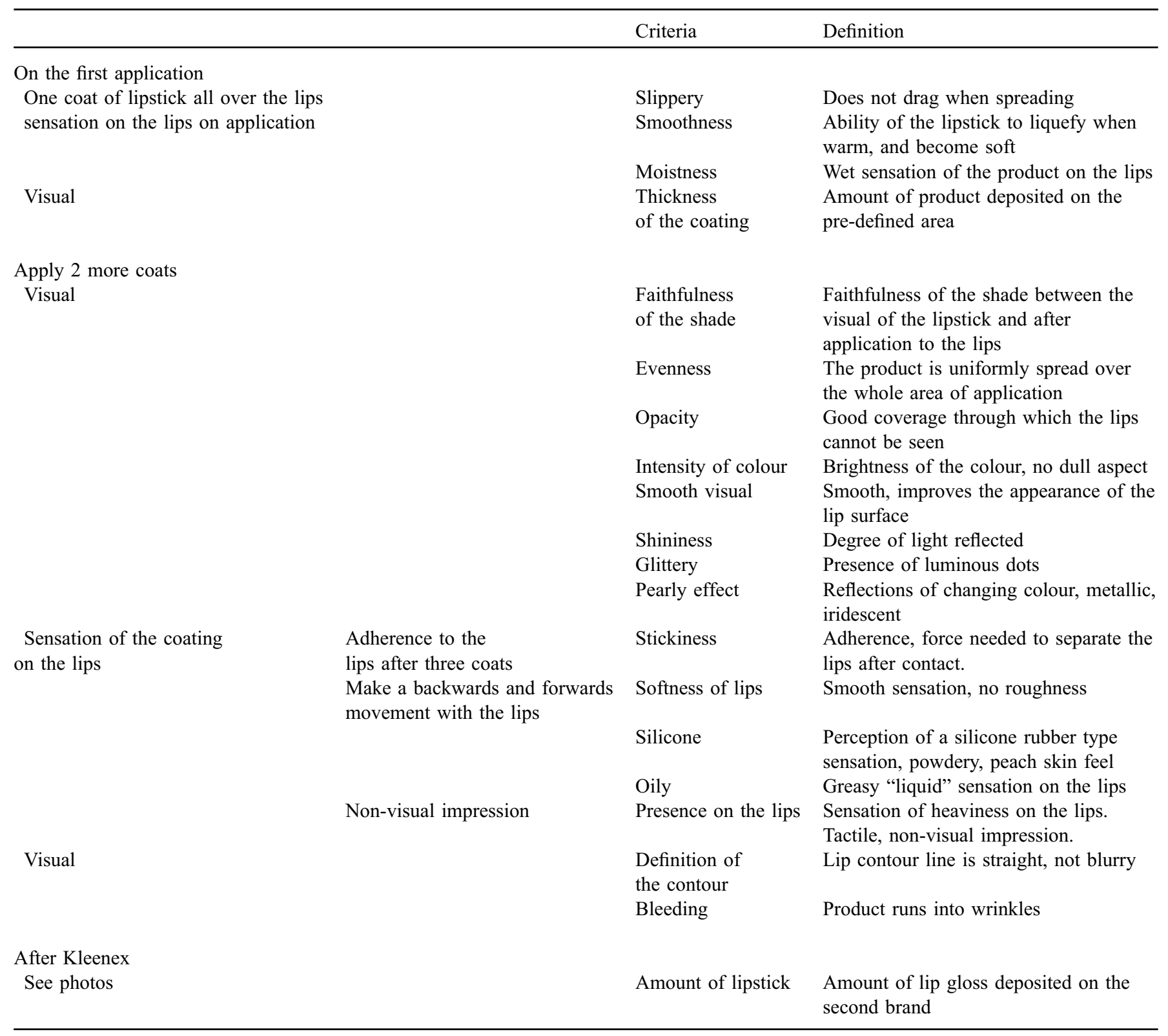

cerebral projection means that the corresponding body part on the sensory homunculus is larger, and the sensory perception is finer. Naturally, the sensory perception of the lips is much greater than on the forearm. The sensory analysis of lipstick on the forearm will therefore not be the same as on the lips.

At Chanel PB, the panel of experts consists of around 20 women trained to assess lip products according to 19 sensory, visual and texture criteria. These 19 criteria, different from those determined by Dooley et al., are assessed on the lips, according to a lip product evaluation protocol that was introduced according to the Afnor standard (AFNOR, 1990). It is a variant of the Spectrum method. This sensory analysis protocol includes the criteria to be assessed and the application procedure. The lipsticks are assessed according to the evaluation protocol presented in Table 2. This protocol was drawn up for all lip products, not only lipstick. Therefore moistness, glitter and a pearly effect are not assessed for lipsticks as these only relate to "lip gloss" products. These sensory criteria are therefore not assessed for lipsticks. Depending on the criterion, the evaluation is carried out according to the perception on the lips or the visual perception. The panel of experts applies the lipstick following a welldefined protocol, in a room where the temperature, lighting and humidity are controlled. The panellists apply the lipstick once, to the lower lip and the upper lip. Three sensory criteria are then assessed: the slipperiness, the smoothness and the thickness. The lipstick is then applied again twice, to the lower lip and the upper lip, to assess the other criteria. Finally, 
Table 3. Oils sensory evaluation protocol on forearm, including the criteria definitions, drawn up by the CHANEL PB sensory analysis department.

\begin{tabular}{|c|c|c|}
\hline & Criteria & Definition \\
\hline When handled & Greasy & $\begin{array}{l}\text { Greasy sensation when rubbing the thumb against the index/middle finger (not greasy - } \\
>\text { very greasy) }\end{array}$ \\
\hline \multirow[t]{3}{*}{$\begin{array}{l}\text { On application } \\
\text { (1 drop) }\end{array}$} & Slipperiness & $\begin{array}{l}\text { Ease of movement of the product on the skin during the first } 4 \text { turns. The product does } \\
\text { not catch in my fingers, it is not resistant to spreading }\end{array}$ \\
\hline & Silicone & Perception of a silicone rubber type feel (Not silicone-> very silicone) \\
\hline & Speed of penetration & $\begin{array}{l}\text { Judge whether the product has disappeared from the skin surface after } 24 \text { turns (slow - } \\
>\text { quick) }\end{array}$ \\
\hline \multirow{4}{*}{$\begin{array}{l}\text { After application } \\
\text { ( } 5 \text { minutes })\end{array}$} & Stickiness & $\begin{array}{l}\text { Adherence, force required to remove the fingers after three contacts with the skin (Not } \\
\text { sticky }->\text { very sticky) }\end{array}$ \\
\hline & Residual grease & Greasy, oily residual film, on the skin surface (not greasy -> very greasy) \\
\hline & Residual silicone & Perception of a silicone rubber type feel (Not silicone-> very silicone) \\
\hline & Residual dryness & After 5 minutes, feeling of chalk, dryness on the fingers (Not dry $->$ very dry) \\
\hline
\end{tabular}

the lipstick is removed from the lips using a paper tissue and the quantity of lipstick applied is assessed.

To assess the new tests, the protocol is initially done with a control. The panellists then memorise the marks they have given for each of the criteria for this control. This is comparable to the "tare" used on scales before weighing a sample. In the case of lipstick, the control used internally is Rouge Allure Shade 09 Lover No. 09. Once the control lipstick has been completely removed, the panellists apply the test lipstick, following the same protocol. The sensory profiles created can be compared to each other over several years, provided that the control stays the same.

A sensory profile of the oils is done following a different protocol from the lipstick protocol. The oils are assessed for their sensory properties, by application to the forearm. In this study, we will focus on the criteria of slipperiness, smoothness, shininess, oiliness and thickness of the application.

As we have previously explained, a sensory assessment of the forearm is not comparable to an assessment on the lips. The sensory properties of the oils cannot be compared to the sensory properties of the lipsticks. However, we can compare the sensory groups of the oils to the sensory groups we obtain for lipsticks. The oils are evaluated according to ten sensory descriptors, defined in Table 3 . Two of the ten criteria evaluated - the speed of penetration of the oils and the emollient nature of the oil - are not relevant for use of the oils in lipsticks. They will therefore not be taken into account in our analysis.

\subsection{Refractive index}

We used an Abbe refractometer from Paralux to measure the refractive index of the oils. The oils are deposited on the glass of the refractometer and measured at $20^{\circ} \mathrm{C}$.

\subsection{Shininess}

The shininess tests for the oils are carried out using the SAMBA from BOSSA NOVA Technologies. A $90 \mu \mathrm{m}$ thick film is spread using a Zehnter universal applicator to the polished black background of a N2A contrast chart from BYK. The chart is dried for 12 hours at $20^{\circ} \mathrm{C}$ before measurement.

\subsection{Viscosity}

The viscosity measurements of the oils, on their own or blended, are carried out with a DHR2 rheometer from TA Instruments. The geometry used is a $4^{\circ} / 40$ cone plate with a gap of $96 \mu \mathrm{m}$. The blended oils, prepared in advance, were then deposited on the lower plate. Different shear values were initially used to confirm that the mixtures follow Newtonian behaviour. The viscosity was then measured at $20^{\circ} \mathrm{C}$.

\subsection{Mechanical properties of lipsticks}

The lipstick bending force was measured using a TA. XTPlus Texture Analyser (Stable Micro Systems) coupled with a lipstick Cantilever Rig. The probe (the lipstick Cantilever Rig) moves down at a constant speed $(5 \mathrm{~mm}$ per second) and bends the lipstick until it breaks. The trigger force is set at $1 \mathrm{~g}$ and the Texture Analyser measures the force according to distance until the lipstick breaks. Measurements are made in triplicate at $20^{\circ} \mathrm{C}$ and the mean of the maximum force measured is reported.

\subsection{PCA}

A Principal Component Analysis was used to construct the sensory charts for the formulated lipsticks. 


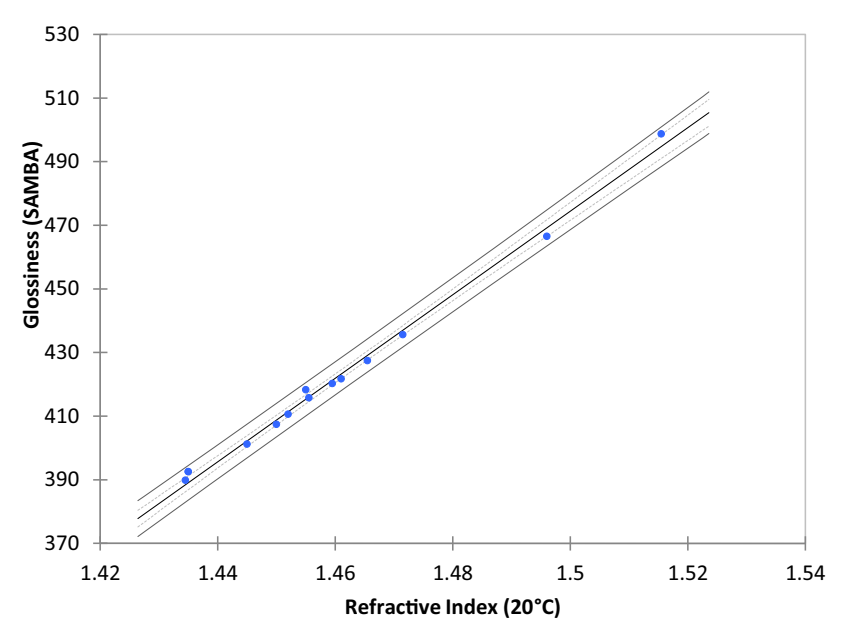

— Modèle(Glossiness SAMBA) Int. de conf. (Obs 95\%)

Fig. 2. Linear regression analysis of Glossiness (Samba) as a function of the Refractive Index $\left(20^{\circ} \mathrm{C}\right)\left(\mathrm{R}^{2}=0.995\right)$.

Correlations between the sensory characteristics of the lipsticks and the physical measurements of the oils were carried out. Correlations between the sensory properties of the lipsticks and their physical properties were established.

\section{Results}

The first relationship we were able to establish concerns the oils on their own. All the physico-chemical parameters measured, such as viscosity, refractive index and shininess, meant we could establish certain relationships (Tab. 1). The viscosity of polybutene could not be measured as this raw material is very viscous. Likewise, the jojoba oil spread on the contrast chart had not dried after 12 hours at $20^{\circ} \mathrm{C}$, so the shininess could not be measured. Based on the measurements performed, there was a direct link between the shininess of the oils and their refractive index (Fig. 2). An excellent correlation exists: the higher the refractive index of the oil, the shinier it is.

By contrast, there was no direct relationship between the refractive index or the shininess and the viscosity of the oils.

The sensory mapping of the oils will determine the proximity between certain raw materials. A PCA of the oils was performed to highlight the correlated sensory criteria (Fig. 3).

In the blue zone, dicaprylyl carbonate and isononyl isononanoate were less greasy between the fingers, slippery on application and quicker to penetrate. The finish was matte, non-sticky, the least greasy, and soft. They were softer, more slippery and more matte. They were also the two oils with the lowest viscosity used in our test plan.

In the green zone, caprylic/capric triglycerides, jojoba oil, squalane, octyl dodecanol, hydrogenated polydecene, meadowfoam seed oil (Limnanthes alba) and pentaerythrityl tetraoctanoate were greasy between the fingers and on the skin, penetrated less quickly and were slippery on application. They were shiny to satiny on the skin, a little sticky and did not leave any dry residue. Some oils from this group were very emollient, particularly squalane. Their viscosity was between 0.035 and 0.140 Pa.s.
Polyglyceryl-2 triisostearate, in the pale pink zone, was greasy between the fingers and on the skin, penetrated relatively quickly and was not too sticky on application.

Finally, phytosteryl/octyldodecanol lauroyl glutamate, polyglyceryl-10 nonaisostearate and diisostearyl malate had extreme sensory properties. These oils are very greasy between the fingers, the least slippery on application, take the longest to penetrate, with a sensation of silicone for phytosteryl/ octyldodecanol lauroyl glutamate in the fuchsia zone. The finish is shiny, the most sticky of the oils tested, very greasy and the least soft. Polyglyceryl-10 nonaisostearate, in the grey zone, is the least dry ester on the skin and the least slippery on application of all the oils tested. These oils have the highest viscosity of those in our test plan, with $2.974 \mathrm{~Pa} . \mathrm{s}$ for phytosteryl/octyldodecanol lauroyl glutamate and 5.302 Pa.s for diisostearyl malate.

Although there is no link between the physico-chemical specifications of shininess and the viscosity of the oils, it is clear that there is a relation between the viscosity of the oils and their sensory properties (Tab. 4). The viscosity of the oils gives variations in sensory properties when applied to the skin. The "slippery" sensory descriptor is therefore greater for more fluid oils. By contrast, the "sticky" descriptor increases when the oil is highly viscous.

Likewise, the more viscous an oil, the more sticky and more greasy it is. Stickiness is in direct opposition to the sensory criterion of softness. Softness, slipperiness and mattness are three very well correlated sensory criteria. On these points, the oils evaluated as the softest, the most slippery and the most matte are also those that are the least viscous.

We can also note that the shininess values of the oils measured in vitro by the Samba (Tab.1) are identical to the classification made in vivo by the sensory analysis panel on the oils evaluated on their own (Fig. 3).

For example, dicaprylyl carbonate and isononyl isononanoate have respective shininess values of 389.8 and 392.5. These oils are evaluated as having a matte to satiny finish. Oils with shininess values between 421 and 435, such as diisostearyl malate, polyglyceryl-2 triisostearate, polyglyceryl-10 nonaisostearate are evaluated as shiny. Between these two extreme groups are oils with intermediate shininess measurements and a sensory evaluation of the finish on the skin as satiny to shiny. The more viscous an oil, the more it is assessed as shiny by the sensory panel. The physico-chemical measurement also helps to give an idea of the sensory evaluation of the raw material on its own, which saves time by avoiding this step. There is in fact no relation between the viscosity measurement of the oils and their shininess measurements. It is therefore interesting to note that the viscosity has a relationship to the "shininess" sensory description of the oil on its own.

It is therefore interesting to see if these sensory properties of the oils on their own still exist once they are formulated as lipsticks. Six lipsticks were formulated using different oils and were evaluated in a sensory analysis. The six oils were selected to represent different sensory characteristics as ingredients, with different viscosities but also different chemical properties. These were esters, a Guerbet alcohol, a vegetable oil and a terpene, in order to highlight the potential impact of the oil's chemical structure within a group of oils of similar viscosity. 


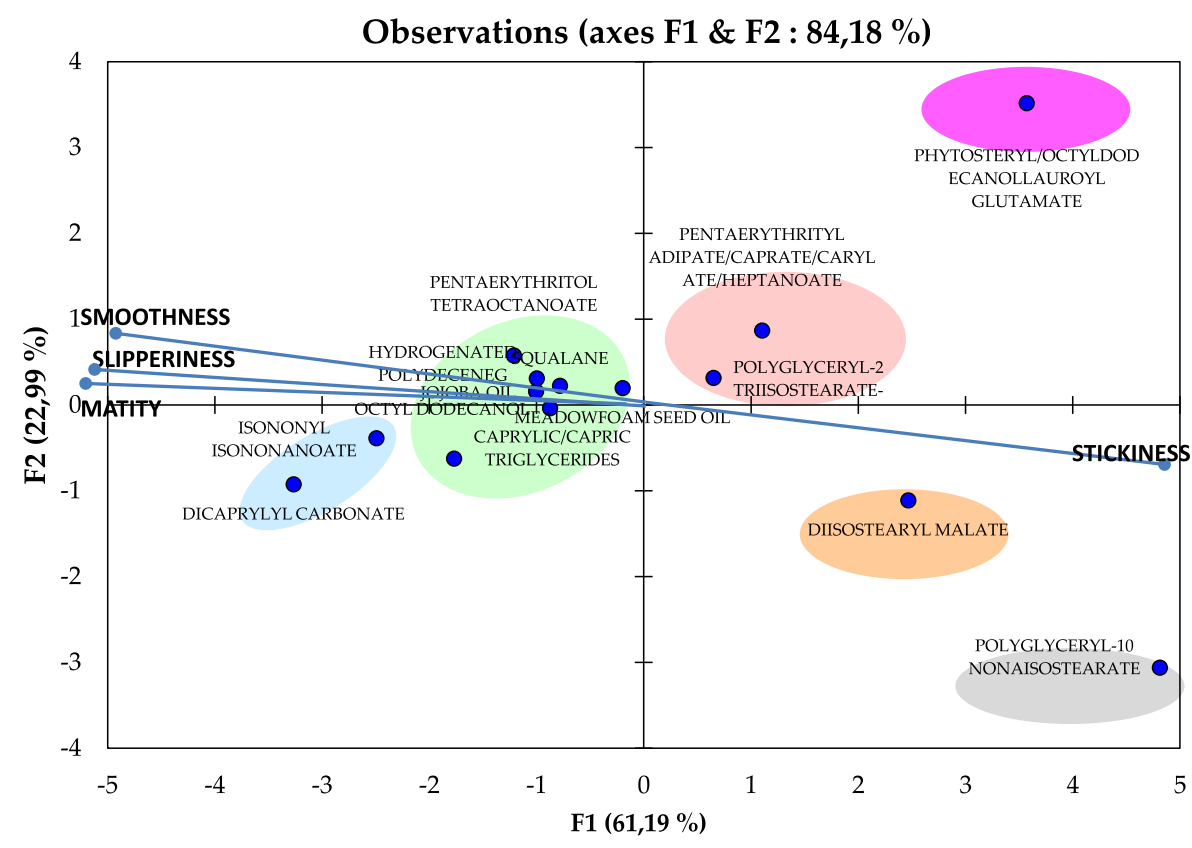

Fig. 3. PCA of the sensorial analysis of the oils including 4 axes of correlation: matity, slipperiness, smoothness and stickiness.

Table 4. Viscosity measurements and classification in sensory groups of oils on their own. The colour of the line corresponds to that of the sensory group of oils on their own, presented in Figure 3.

\begin{tabular}{|c|c|c|}
\hline OILS & Viscosity Pa.s $\left(20^{\circ} \mathrm{C}\right)$ & Sensorial group \\
\hline ISONONYL ISONONANOATE & $0.009 \pm 0.00006$ & Very extreme: the least greasy on the fingers, not very slippery on application and the quickest to penetrate. Leaves the skin the most \\
\hline DICAPRYLYL CARBONATE & $0.0105 \pm 0.00004$ & matte, very slightly sticky, non-greasy, slightly soft, with a slight sensation of dryness/talc and the most emollient. \\
\hline CAPRYLIC/CAPRIC TRIGLYCERIDE & $0.035 \pm 0.0001$ & \multirow{7}{*}{$\begin{array}{l}\text { Greasy to not very greasy, penetrates fairly quickly, leaving the skin satiny to matte, slightly sticky, slightly t greasy to non-greasy and } \\
\text { slightly emollient. }\end{array}$} \\
\hline JOJOBA OIL & $0.043 \pm 0.0006$ & \\
\hline SQUALANE & $0.049 \pm 0.0001$ & \\
\hline OCTYL DODECANOL & $0.058 \pm 0.0004$ & \\
\hline HYDROGENATED POLYDECENE & $0.070 \pm 0.0001$ & \\
\hline MEADOWFOAM SEED OIL (LIMNANTHES ALBA) & $0.104 \pm 0.002$ & \\
\hline \begin{tabular}{|l} 
PENTAERYTHRITYL TETRAOCTANOATE \\
\end{tabular} & $0.140 \pm 0.002$ & \\
\hline POLYGLYCERYL-2 TRIIISOSTEARATE & $0.549 \pm 0.003$ & Quite greasy on the fingers and the skin, penetrates less quickly, very emollient, leaves the skin a little shiny \\
\hline POLYGLYCERYL-10 NONAISOSTEARATE & $1.839 \pm 0.012$ & $\begin{array}{l}\text { The most greasy, takes a long time to penetrate, with a sensation of silicone. The finish is the shiniest, very sticky, the most greasy and the } \\
\text { most emollient. }\end{array}$ \\
\hline PHYTOSTERYL/OCTYLDODECANOL LAUROYL GLUTAMATE & $2.974 \pm 0.034$ & $\begin{array}{l}\text { Quite extreme: very greasy between the fingers, the least slippery, takes a long time to penetrate, with a sensation of silicone for } \\
\text { Phytosteryl/ Octyldodecanol Lauroyl Glutamate. The finish is shiny, the most sticky, very greasy and the least soft. }\end{array}$ \\
\hline DIISOSTEARYL MALATE & $5.302 \pm 0.028$ & $\begin{array}{l}\text { Very greasy on the fingers and the skin, penetrates less quickly, leaves the skin a little shiny to satiny, slightly sticky and no dry } \\
\text { residue/talc. }\end{array}$ \\
\hline
\end{tabular}

Six lipsticks were made with a similar formula, each using a different oil.

Figure 4 shows the positioning of the lipsticks in PCA after sensory evaluation by the panel. We can see different coloured zones. Each represents the space in which the sensory properties are not significantly different. All the lipsticks in a defined coloured zone are judged to be similar from a sensory point of view.

The lipsticks formulated with dicaprylyl carbonate and isononyl isononanoate are in the blue zone. These are the most matte, most slippery and least sticky lipsticks. We therefore find the specific properties already identified for these two oils as raw materials on their own.

By contrast, phytosteryl/octyldodecanol lauroyl glutamate is one of the most viscous oils in the study. Logically, lipsticks made with this oil have a strong correlation with the stickiness descriptor. They are in the pink zone.
Likewise, squalane, octyl dodecanol and meadowfoam seed oil (Limnanthes alba), which are in the same sensory group as oils on their own, result in lipsticks in the same green sensory zone on Figure 4. This is even more interesting as, in addition to the fact that the sensory properties of the oils on their own are similar, the chemical natures of these oils are very different. We can deduce from this experience that some sensory characteristics of the oils on their own are also found in the lipsticks. However, the focus on which the positioning of the six lipsticks is organised is primarily based on the viscosity. The chemical nature of the oil is not a factor, or not a significant factor.

For the oils on their own, the sensory properties are mainly influenced by the viscosity of the oil. This parameter is also significant for the formulated lipsticks: when the most slippery oils are made into lipsticks, they result in the most slippery lipsticks. The more fluid the oil, the more slippery the lipstick. 


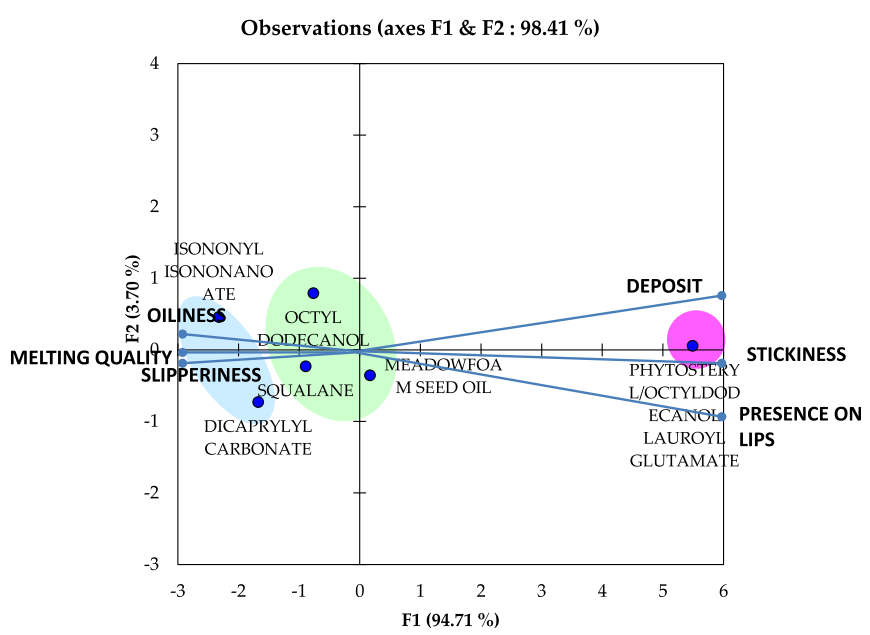

Fig. 4. PCA of the sensorial analysis of the lipsticks formulated with a different oil including the areas of correlation: slipperiness and stickiness. Each colour corresponds to a zone in which the sensory properties of the lipsticks are not significantly different.

Likewise, the more viscous an oil, the more sticky it is. Lipstick made with this oil is sticky and the thickness of the coating on the lips on application is significant. As a result, the sensation of presence on the lips is strong. All these descriptors are strongly correlated. In Figure 4, new sensory descriptors appear, generated by the lipstick and not by the raw material on its own.

A surprising point is that the shininess of the lipsticks does not seem to be correlated to the shininess or the refractive index of the pure oils. If that was the case, the "Matte" sensory descriptor would be correlated to the lipsticks formulated with dicaprylyl carbonate and isononyl isononanoate. However, this descriptor does not appear on the PCA. It is not correlated to any previously defined category of oils. Although oils on their own can be classified according to shininess, which is itself strongly correlated to the refractive index, this sensory descriptor is not significant for lipsticks. Therefore, the shininess of the oils does not influence the shininess of the lipsticks and the refractive index measurement of the oils does not help to predict the shininess of the lipstick. Shininess in the formulation is therefore provided by something other than the selection of oils with high refractive indices. One of the hypotheses is that the shininess caused by the specular reflection of light on a smooth surface, is dependent on the quality of the coating on the lips. It could be that a very smooth, even coating of constant thickness results in a greater specular reflection than a coating that is rough on the surface. To achieve this, the waxes must be crystallised uniformly and not contain any microcrystals. We are not going to deal here with the impact of the process on wax crystallisation and on the quality of the coating obtained (AFNOR, 1990). We worked using a constant process for all the tests. However, the pouring temperature and the cooling ramp are the predominant criteria in the crystalline structure obtained at the end of the process (Cansell and Talence, 2005). Other parameters may also impact the shininess of a wax-oil blend (Snabre and Habouzit, 2013). The solubility of the wax with the oil in the formula determines the shapes and sizes of the crystals that form in the stick. We also thought it would be a good idea to create new

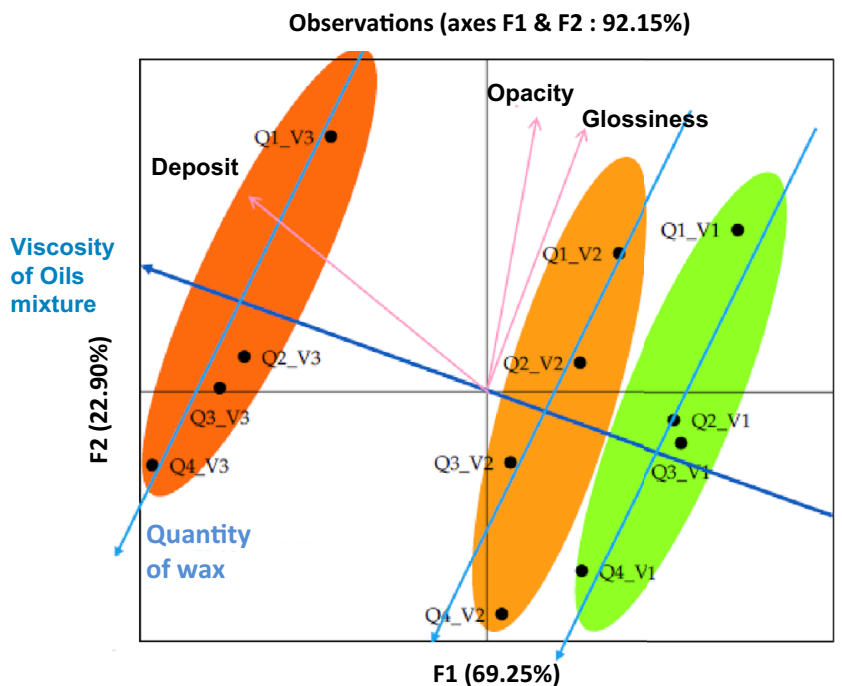

Fig. 5. Influence of the chemical nature of the wax, its concentration and the viscosity of oils in the formula on the sensory descriptors. Each coloured zone corresponds to the same chemical nature of wax tested at four different concentrations. Q1 is the lowest concentration, Q4, the highest. V1 is the oil with the lowest viscosity. V3 is the most viscous oil.

lipstick formulas by varying the chemical nature of the waxes. Three waxes were tested at four different concentrations (Q1 the lowest and Q4 the highest). Three oils were selected for their distinct viscosities (from V1 the lowest to V3 the highest): dicaprylyl carbonate, meadowfoam seed oil (Limnanthes alba) and phytosteryl/octyldodecanol lauroyl glutamate. Twelve formulas were made with the three waxes, four concentrations and three oils with different viscosities (Fig. 5). On the PCA, the lipsticks made with the oil with a low viscosity are in the green zone. These are the ones that deposit the thinnest coating on the lips. The lipsticks made with the oil with an intermediate viscosity are in the orange zone. Those made with the most viscous oil are correlated with the thickness sensory descriptor. The more viscous the oil used in the formula, the thicker the lipstick coating.

The wax concentration is also correlated with the sensory descriptors, but different to those relating to the viscosity of the oils. In the case of the lowest wax concentrations (Q1), the panel evaluated the lipsticks as being the most opaque and the shiniest. They also left more of a coating on the lips. This was more marked for the wax in the red zone, which had a tendency to deposit more lipstick than the other two. The lipsticks with the highest quantity of wax (Q4) are less opaque, deposit less of a coating and are less shiny.

The type of wax also has an impact on the sensory nature of the lipsticks. The three coloured zones are distinct and do not overlap, which shows that wax is one of the factors that can alter the position of a lipstick within a sensory chart. In addition, the wax in the red zone leaves more of a coating on the lips.

Our hypothesis for interpreting these results is as follows: the lipstick with the lowest concentration of wax is softer and creamier, less solid than the one with a higher concentration. It therefore deposits more of a coating, which makes it more opaque. Finally, a lower wax concentration has fewer crystals 


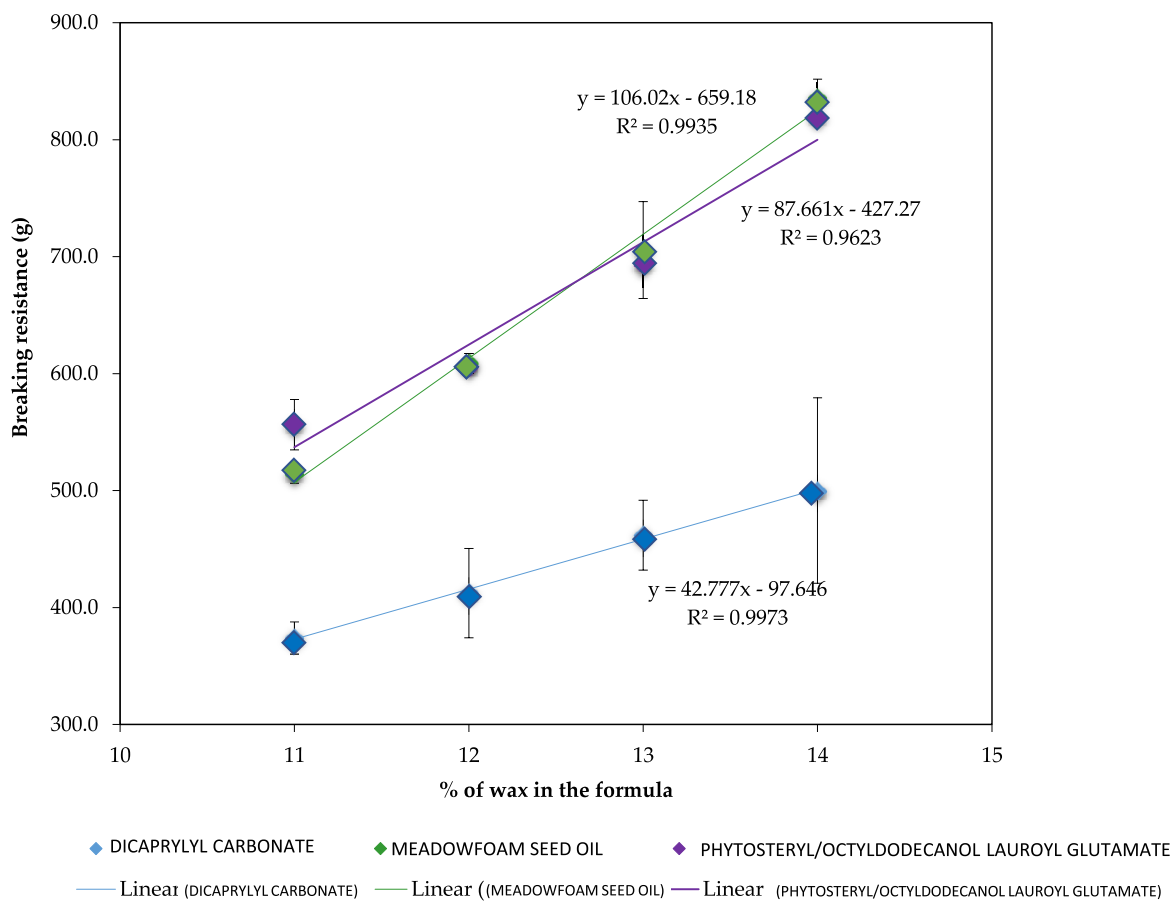

Fig. 6. Breaking resistance of lipsticks as a function of the quantity of wax in the formula for three oils from different viscosities.

in the formula. The lipstick has a smoother texture. The coating is more uniform and the specular light is better reflected, which increases the shininess.

Finally, the quality of the lipsticks was evaluated. One of the most important quality measurements was the resistance to breaking of the lipstick. This value was systematically verified, as women use a force of 500 to 600 Newtons to lipstick when they apply it (de Carames, 1978; Drew, 1978). This is why controls of mechanical properties are strategic and brands fix an acceptability threshold close to 600 Newtons in their physico-chemical specifications. It would be inconceivable for a luxury brand to sell a lipstick that would break on first use. We therefore studied lipsticks with oils of different viscosities. Dicaprylyl carbonate, which is the least viscous oil, meadowfoam seed oil (Limnanthes alba), which is an intermediate viscous oil, and phytosteryl/octyldodecanol lauroyl glutamate, which is one of the most viscous oils. Four concentrations of synthetic wax \& polyethylene \& polypropylene/ethylene copolymer were examined with each of these oils: 11, 12, 13 and 14\% (Fig. 6).

The first observation was that the greater the wax concentration, the greater the lipstick's resistance to breaking. For a wax concentration of $11 \%$, the resistance to breaking measurements were between $370 \mathrm{~g}$ and $560 \mathrm{~g}$. These were nonstandard values from a quality point of view. By contrast, for a wax concentration of $14 \%$, the resistance to breaking was between $495 \mathrm{~g}$ and $840 \mathrm{~g}$, which made the products formulated with the two most viscous oils acceptable from a quality point of view.

In addition, the curve of the linear relation between resistance to breaking and the percentage of wax in the formula varies according to the viscosity of the oil. The more viscous the oil, the greater the resistance to breaking.
The increase in the viscosity of the oil also works in the same way as the increase in wax concentration on the resistance to breaking of the lipsticks. This is interesting for formulators, as we have previously seen that a greater amount of wax increases the mattness of the lipstick. If it is necessary to reinforce the resistance to breaking of a shiny lipstick, the only method available to formulators is not to add wax to the formula. There is an alternative which is increasing the viscosity of the oils present in the blend. This will help to maintain shininess while improving mechanical performance.

\section{Conclusion}

The objective of this study is to be able to predict the sensory properties of lipsticks from the physico-chemical specifications of the ingredients or raw materials. The viscosity of oils is the specification which has the most impact on the sensory properties of lipsticks: slipperiness, thickness of coating, stickiness, smoothness. The viscosity of oils also influences the resistance to breaking of lipsticks. This physicochemical parameter is also essential for modifying the texture of lipsticks. Wax also plays an important role in the sensory properties of lipsticks, but the sensory descriptors used are often different from those influenced by the viscosity of the oils. Waxes impact the opaqueness, shininess and thickness of the coating. They of course enable the mechanical properties of lipsticks to be adjusted.

Oils with low viscosity increase the slipperiness of a lipstick, whereas oils with high viscosity increase the thickness of the coating. The resistance to breaking can be increased by selecting oils with high viscosity or by increasing the percentage of wax in the formula. The oil-wax blend is very important, as it determines the number and size of the crystals 
present in the texture, which also affects the sensory properties, such as the shininess, and the mechanical properties of the lipstick. This knowledge means we can investigate new sensory territories or reduce the time-to-market of products in development without compromising the quality of the products offered to consumers in any way.

\section{References}

Abidh S. 2017. Méthodologie d'ingénierie sensorielle pour la formulation de produits cosmétiques, application au rouge à lèvres. Thèse de Doctorat NNT: 2017SACLA023.

Abidh S, Cuvelier G, de Clermont-Gallerande H, Navarro S, Delarue J. 2016. Sensory-designed lipsticks: from sensory needs to raw materials selection, a new way of formulation, Proceeding of the 29th IFSCC Congress, 31st Oct.-2nd Nov. 2016, Orlando, FL.

AFNOR. 1990. Analyse sensorielle-recherche et sélection de descripteurs pour l'élaboration d'un profil sensoriel, par approche multidimensionnelle. NF V09-021 mai 1990.

Cansell M, Talence FF. 2005. Impact de la cristallisation des corps gras sur les propriétés des produits finis. OCL 12: 427-443.

de Carames, M. 1978. Development of a lipstick base. Cosmet Toil 93: 15.

de Clermont-Gallerande H. 2006. Évolution des corps gras utilisés dans la formulation des rouges à lèvres au cours des 15 dernières années. $O C L$ 13: 322-325.

Desmet P, Hekkert P. 2007. Framework of Product Experience. Int $J$ Des 1(1): 13-23.

Dooley LM, Adhikari K, Chambers IV.E. 2009. A general lexicon for sensory analysis of texture and appearance of lip products. J Sens Stud 24(4): 581-600.
Drew RC. 1978. Evaluation of mechanical stresses set up in lipsticks during application. J SOC Cosmet Chem 29: 441.

Hill SE, Rodeheffer CD, Griskevicius V, Durante K, White AE. 2012. Boosting beauty in an economic decline: mating, spending, and the lipstick effect. J Personal Soc Psychol 103(2): 275-291.

Martinez A, Allison M. 2010. Beauty-products sales bright spot during recession. The Seattle Times, 09th September 2010.

Meilgaard MC, Civille GV, Carr BT. 2006. Sensory evaluation techniques. Boca Raton, FL: CRC Press.

Nelson E. 2001. Rising lipstick sales may mean pouting economy and few smiles. The Wall Street Journal, 26th November 2001.

Oglivie M, Kristensen-Bach P. 2015. Why women wear lispticks: preliminary findings. Available from https://www.researchgate. net/publication/49282703_Why_women_wear_lipstick_prelimi nary findings. (last consult 2018/02/02).

Pensé-Lhéritier AM. 2015. Recent developments in the sensorial assessment of cosmetic products: A review. Int J Cosmetic Sci 37 (5): 465-473.

Russell R, Porcheron A, Sweda JR, Jones AL, Mauger E, Morizot F. 2016. Facial contrast is a cue for perceiving health from the face. $J$ Exp Psychol: Human Percept Perform 42(9): 1354-1362.

Savary G, Grisel M, Picard C. 2013. Impact of emollients on the spreading properties of cosmetic products: a combined sensory and instrumental characterization. Colloids Surf B: Biointerfaces 102: $371-378$.

Schaefer K. 2008. Hard times, but your lips look great. The New York Times, 01st May 2008.

Snabre P, Habouzit D. 2013. Chauffage, endommagement et exsudation des matériaux semi-cristallins à base de corps gras. I-Revues, 21 ${ }^{\mathrm{e}}$ Congrés Français de Mécanique [CFM2013], Colloques thématiques, $\mathrm{C} 10-$ Rencontres physique/mécanique.

Cite this article as: de Clermont-Gallerande H, Abidh S, Lauer A, Navarro S, Cuvelier G, Delarue J. 2018. Relations between the sensory properties and fat ingredients of lipsticks. OCL 25(5): D502. 